\title{
Oxford Nanopore MinION Sequencing Enables Rapid Whole Genome Assembly of Rickettsia typhi in a Resource-Limited Setting
}

\author{
Ivo Elliott, ${ }^{1,2 *} \dagger$ Elizabeth M. Batty, ${ }^{2,3,4} \dagger$ Damien Ming, ${ }^{1}$ Matthew T. Robinson,,${ }^{1,2}$ Pruksa Nawtaisong, ${ }^{1}$ Mariateresa de Cesare, ${ }^{3}$ \\ Paul N. Newton, ${ }^{1,2} \ddagger$ and Rory Bowden ${ }^{3} \ddagger$ \\ ${ }^{1}$ Lao-Oxford-Mahosot Hospital-Wellcome Trust Research Unit, Microbiology Laboratory, Mahosot Hospital, Vientiane, Lao PDR; ${ }^{2}$ Nuffield \\ Department of Medicine, Centre for Tropical Medicine and Global Health, University of Oxford, Oxford, United Kingdom; ${ }^{3}$ Wellcome Centre for \\ Human Genetics, University of Oxford, Oxford, United Kingdom; ${ }^{4}$ Mahidol-Oxford Tropical Medicine Research Unit, Faculty of Tropical Medicine, \\ Mahidol University, Bangkok, Thailand
}

\begin{abstract}
The infrastructure challenges and costs of next-generation sequencing have been largely overcome, for many sequencing applications, by Oxford Nanopore Technologies' portable MinION sequencer. However, the question remains open whether MinION-based bacterial whole genome sequencing is by itself sufficient for the accurate assessment of phylogenetic and epidemiological relationships between isolates and whether such tasks can be undertaken in resource-limited settings. To investigate this question, we sequenced the genome of an isolate of Rickettsia typhi, an important and neglected cause of fever across much of the tropics and subtropics, for which only three genomic sequences previously existed. We prepared and sequenced libraries on a MinION in Vientiane, Lao PDR, using v9.5 chemistry, and in parallel, we sequenced the same isolate on the Illumina platform in a genomics laboratory in the United Kingdom. The MinION sequence reads yielded a single contiguous assembly, in which the addition of Illumina data revealed 226 base-substitution and 5,856 indel errors. The combined assembly represents the first complete genome sequence of a human $R$. typhi isolate collected in the last 50 years and differed from the genomes of existing strains collected over a 90 -year time period at very few sites, with no rearrangements. Filtering based on the known error profile of MinION data improved the accuracy of the nanopore-only assembly. However, the frequency of false-positive errors remained greater than true sequence divergence from recorded sequences. Although nanopore-only sequencing cannot yet recover phylogenetic signals in $R$. typhi, such an approach may be applicable for more diverse organisms.
\end{abstract}

\section{INTRODUCTION}

Until recently, whole genome sequencing (WGS) has been the preserve of high-income settings. Although the costs of WGS have dramatically decreased over the past decade, ${ }^{1}$ the need for initial investment in sequencing platforms and associated equipment and for the technical expertise to run and maintain them have prevented their introduction into lower income settings. Downstream processing of sequencing data is frequently hampered by poor Internet connectivity, a lack of local expertise, and the perceived requirement for substantial computational infrastructure.

Oxford Nanopore Technologies' (ONT) MinION sequencer is a portable device for DNA and RNA sequencing that generates data for local analysis, in real time. The MinION weighs under $100 \mathrm{~g}$ and plugs directly into a laptop via a USB port, with no additional computing infrastructure required. ${ }^{2}$ Only basic laboratory facilities are needed to extract DNA and prepare sequencing libraries. The MinION has been used to sequence viral, ${ }^{3}$ bacterial, ${ }^{4,5}$ and eukaryotic ${ }^{6-9}$ genomes. Despite its high raw error rate, nanopore data can in many cases produce highly contiguous assemblies. Illumina short read data can be added to improve consensus accuracy, in many cases to that of a high-quality draft assembly, without extra finishing steps.

The Lao People's Democratic Republic (Laos) is a land-linked country of $\sim 7$ million people in Southeast Asia. Lao People's Democratic Republic is ranked 135th in the world on the Human Development Index, a composite statistic of life expectancy, education, and per capita income. ${ }^{10}$ There are few functioning

*Address correspondence to Ivo Elliott, Lao-Oxford-Mahosot Hospital Wellcome Trust Research Unit, Microbiology Laboratory, Mahosot Hospital, Vientiane, Lao PDR. E-mail: ivo@tropmedres.ac

†These authors contributed equally to this work.

$\ddagger$ These authors contributed equally to this work. molecular biology laboratories in Laos, no previous published WGS projects, and relatively poor Internet connectivity. ${ }^{11}$

Rickettsia typhi is an obligate intracellular, Gram-negative bacterium in the family Rickettsiaceae that causes the disease murine typhus and has a worldwide tropical and subtropical distribution. ${ }^{12,13}$ The pathogen is transmitted to humans primarily by flea fecal contamination of the bite of the oriental rat flea Xenopsylla cheopis. ${ }^{14}$ The disease is an important and grossly under-recognized global cause of febrile illness. ${ }^{15}$ Those affected suffer from high fever, headache, myalgia, arthralgia, nausea, and vomiting and may have a macular rash. Complications are infrequent but include myocarditis, meningoencephalitis, seizures, and renal failure. ${ }^{13,16}$ When diagnosed, recovery is typically rapid after treatment with doxycycline and mortality is low (around 1-2\%) with antibiotic treatment. ${ }^{12,17}$

Despite its importance as a pathogen, to date just three $R$. typhi whole genome sequences, with wide geographic and temporal distribution, have been published. The type strain Wilmington was isolated from a patient in North Carolina in 1928, and the sequence was published in 2004 . $^{18,19}$ Two further sequences originating from a patient in Northern Thailand collected in 1965 and the other from a bandicoot rat, Bandicota sp., collected in Burma (now Myanmar) in the $1970 s^{20-22}$ were published online in 2014 (Table 1).

We report the successful WGS of a human isolate of $R$. typhi using only the MinION platform in Laos. We assess the potential for use of ONT data alone to perform comparative analyses and discuss the challenges of undertaking WGS in resource-limited settings.

\section{MATERIALS AND METHODS}

Rickettsia typhi culture and DNA extraction. Frozen L929 mouse fibroblast cells (ATCC CCL-1), infected with $R$. typhi isolated from the blood of a patient, TM2540, from Pakkading 
TABLE 1

Strain information

\begin{tabular}{|c|c|c|c|c|c|}
\hline Strain & Source & Accession & Genome length (bp) & Predicted genes & GC percentage \\
\hline Wilmington & Human, North Carolina, USA. 1928 & NC_006142.1 & $1,111,496$ & 865 & 0.289 \\
\hline B9991CWPP & Bandicoot rat, Myanmar. 1970 & NC_017062.1 & $1,112,957$ & 865 & 0.289 \\
\hline TH1527 & Human, Chiang Rai, Thailand. 1965 & NC_017066.1 & $1,112,372$ & 865 & 0.289 \\
\hline TM2540 & Human, Bolikhamxay, Laos. 2012 & ERZ497871 & $1,111,939$ & 866 & 0.289 \\
\hline
\end{tabular}

district $\left(18.33^{\circ} \mathrm{N} 104.0^{\circ} \mathrm{E}\right)$, Bolikhamxay Province, Laos, with suspected acute murine typhus infection presenting in 2012 to Mahosot Hospital, Vientiane, were reanimated. Frozen aliquots were briefly thawed at room temperature and then transferred to $25-\mathrm{cm}^{2}$ cell culture flasks containing an L929 cell monolayer at $80 \%$ confluence in RPMI 1640 medium (Gibco, Thermo Fisher, Waltham, MA), supplemented with $10 \%$ fetal calf serum (Sigma-Aldrich, St. Louis, MO). Flasks were incubated at $35^{\circ} \mathrm{C}$ in $5 \% \mathrm{CO}_{2}$ atmosphere for 7 days, and a fraction of cells were mechanically detached and $3 \mathrm{~mL}$ transferred to a new $75-\mathrm{cm}^{2}$ flask containing an L929 monolayer and incubated for a further 7 days in the same conditions.

For DNA extraction, cells from three $75-\mathrm{cm}^{2}$ flasks were mechanically detached, resuspended, and transferred to a 50$\mathrm{mL}$ conical-bottom centrifuge tube and centrifuged at 3,220 $\times \mathrm{g}$ for 10 minutes. The pellet was resuspended in $3-\mathrm{mL}$ fresh medium and transferred to $1.5 \mathrm{~mL}$ microcentrifuge tubes. Tubes were vortexed for one minute and centrifuged at $300 \times \mathrm{g}$ for 3 minutes. The supernatant was passed through a $2-\mu \mathrm{m}$ filter (Corning, Corning, NY), and DNAase was added to the filtrate to a final concentration of $14 \mu \mathrm{g} / \mathrm{mL}$ and, then, incubated at room temperature for 30 minutes. The mixture was centrifuged at $18,188 \times \mathrm{g}$ for 10 minutes, and the pellet washed twice with $0.3 \mathrm{M}$ sucrose (Sigma-Aldrich). Then, DNA was extracted from the $R$. typhi pellet using the DNeasy Blood \& Tissue kit (Qiagen, Hilden, Germany) following the manufacturer's protocol. Then, DNA was eluted in AE buffer and stored immediately at $-20^{\circ} \mathrm{C}$.

The eluted DNA was quantified using Qubit dsDNA High Sensitivity assay kit (Thermofisher, Waltham, MA) following the manufacturer's protocols and assayed for $R$. typhi sequences by the quantitative polymerase chain reaction targeting the $17-\mathrm{kDa}$ outer membrane antigen. ${ }^{23}$

Preparation of MinION sequencing libraries. The MinION sequencing library was produced using the ONT 1D genomic DNA by following the ligation (SQK-LSK108) protocol. In brief, $1.4 \mu \mathrm{g}$ of DNA was fragmented in a Covaris g-TUBE (Covaris Ltd., Brighton, United Kingdom) by centrifugation to produce fragments of $8 \mathrm{~kb}$ in length. Sheared DNA was repaired using NEBNext FFPE repair mix (New England Biolabs, Ipswich, $M A)$. End-repair and dA-tailing were performed with the NEBNext Ultra II End Repair/dA-Tailing module. Adapter ligation used the NEB Blunt/TA Ligase Master Mix, and the library was purified using Agencourt AMPure XP beads (Beckman Coulter Inc., Brea, CA).

MinION sequencing in Laos. MinION libraries were sequenced for 48 hours on an ONT MinION R9.5 flow cell, connected to a Dell Latitude E5470 XCTO laptop with 256 GB SATA Class 20 solid state drive. Oxford Nanopore Technologies fast5 data files were base-called using the ONT Albacore module v2.2.7.

Illumina sequencing in Oxford. An Illumina sequencing library was generated from the same sample of $R$. typhi DNA using the Nextera XT (Illumina) library preparation method. The library was sequenced on the Illumina MiSeq with $2 \times 250$ bp reads. A total of 2,402,136 read pairs were sequenced, giving 1.2 Gbp of total sequence data.

Bioinformatic analysis. The species composition of the MinION reads was identified using Centrifuge v1.0.3 software $^{24}$ testing against the prebuilt nonredundant database and keeping only the best hit per read.

Assembly strategies that used MinION data alone, or in combination with Illumina short reads, were assessed. A draft genome assembly was generated from the MinION reads using Canu v1.4 ${ }^{25}$ with the suggested parameters for ONT sequencing "correctedErrorRate $=0.120$-nanopore-raw" and an estimated genome size of $1.1 \mathrm{Mb}$ and polished using Nanopolish v0.10.2. ${ }^{4}$ The Illumina reads were mapped to the Canu+Nanopolish assembly using bwa $0.7 .12,{ }^{26}$ and the mapped reads were used to error-correct the assembly using Pilon $v 1.22^{27}$. To check for enrichment of errors in regions of low coverage, the ONT reads were mapped onto the corrected assembly using bwa mem to determine coverage, and the coverage at error positions was compared with the total coverage distribution. To look for enrichment of errors near homopolymers, we compared the distance with the nearest homopolymer of $5 \mathrm{bp}$ or longer in length for each corrected error to a random sample of positions in the genome. To assess the gene content, BUSCO (Benchmarking Universal Single-Copy Orthologs) v3 using the OrthoDB v9 bacterial data set was used. ${ }^{28}$ The Illumina short reads alone were assembled using Unicycler. ${ }^{29}$ Prokka $v 1.14^{30}$ was used to annotate both the new assemblies and the existing $R$. typhi genomes to give consistent data for comparison. The Wilmington reference strain was used to train the gene model for the Prodigal gene prediction used in Prokka, and this training set was used to annotate the other three genomes. The new genome was rotated to begin with the yqfL gene for consistency with other $R$. typhi genomes. Roary $v 3.12 .0$ was used to determine the core and accessory genomes. ${ }^{31}$

Data availability. The three existing $R$. typhi genomes were downloaded from RefSeq under the accession numbers NC_ 006142.1, NC_017066.1, and NC_017062.1. Gene identifiers are given as the locus tags in these annotations. The sequence data are available at the ENA under project PRJEB2567. The new strain is named TM2540, and the assembly (final errorcorrected version) is available at the ENA under accession number ERZ497871.

\section{RESULTS}

The MinION flow cell and reagents were shipped from Oxford, United Kingdom, to Laos at $+4^{\circ} \mathrm{C}$. Flow cell quality control before dispatch recorded 1,242 active pores and on receipt after 72 hours travel, 1,103 active pores were recorded as available. Sequencing using the MinKNOW platform was performed for 48 hours and generated approximately 250,000 
reads, with a total fast 5 file size of $20 \mathrm{~GB}$. In total, 222,848 reads passed quality filters and were used in further analysis.

We assessed the species composition of the reads that passed quality filters. Because the $R$. typhi sample was grown in L929 fibroblast cells, we expected to see some contaminating reads from the mouse genome. In all, 134,802 (60\%) of the reads were classified as belonging to the genus Rickettsia, 126,447 reads were classified as $R$. typhi at the species level, and $41,428(19 \%)$ were classified as belonging to the genus Mus (Figure 1).

The FASTQ files produced by Albacore were assembled using Canu, an assembler designed for noisy single-molecule sequencing such as that produced by the MinION. This produced a single-contig assembly of 1,078,916 bp. Nanopolish, a tool to use the signal-level data from Oxford Nanopore sequencing, was used to improve the consensus sequence of the Canu assembly, producing a new assembly of $1,099,322 \mathrm{bp}$. To further correct errors in the assembly produced from long reads alone, we polished the Canu + Nanopolish genome assembly using Pilon, which uses short read sequencing data to correct errors. We repeated rounds of mapping and correction with Pilon until no further errors were corrected. After four rounds of correction, Pilon did not correct any further errors, with the exception of two short indels which were removed and then reinserted by successive rounds of polishing. In total, $94.2 \%$ of errors were corrected by the first round of polishing, with a further $4.8 \%$ corrected in the second round (Supplemental Table 1).

After this process, a total of 6,082 errors were corrected, with over $95 \%(5,856)$ being small insertions and deletions, with the result that $12 \mathrm{~kb}$ was added to the genome during polishing for a final length of 1,111,939 bp. In comparison, when Pilon was run on the Canu draft genome before Nanopolish polishing, 19,970 errors were corrected, confirming that Nanopolish improves the draft genome.

We assessed the ONT read coverage and proximity to a homopolymer run (five bases or longer) for each of the errors (both single-nucleotide polymorphism [SNP] and indel) we corrected with Pilon. We observed no difference in the coverage distribution at sites with errors compared with the overall coverage distribution (Supplemental Figure 2). However, the positions around homopolymer runs were enriched for errors (two-tailed Kolmogorov-Smirnov test statistic $=0.019, P=$ 0.035 ) (Figure 2). Whereas $15 \%$ of the final polished genome is within 5 bp of a homopolymer run (165 kb), 43\% of errors $(2,641)$ are within $5 \mathrm{bp}$ of a homopolymer run.

The Illumina data alone were assembled using Unicycler to compare with assemblies using ONT only and the combined ONT and Illumina data. The assembly generated by Unicycler had 29 contigs, with a total length of 1,161,002 bp and an n50 of $166 \mathrm{~kb}$. However, this included 20 short contigs under $6 \mathrm{~kb}$, which are closely related to the mouse genome by BLAST

${ }^{167}$ Archaea

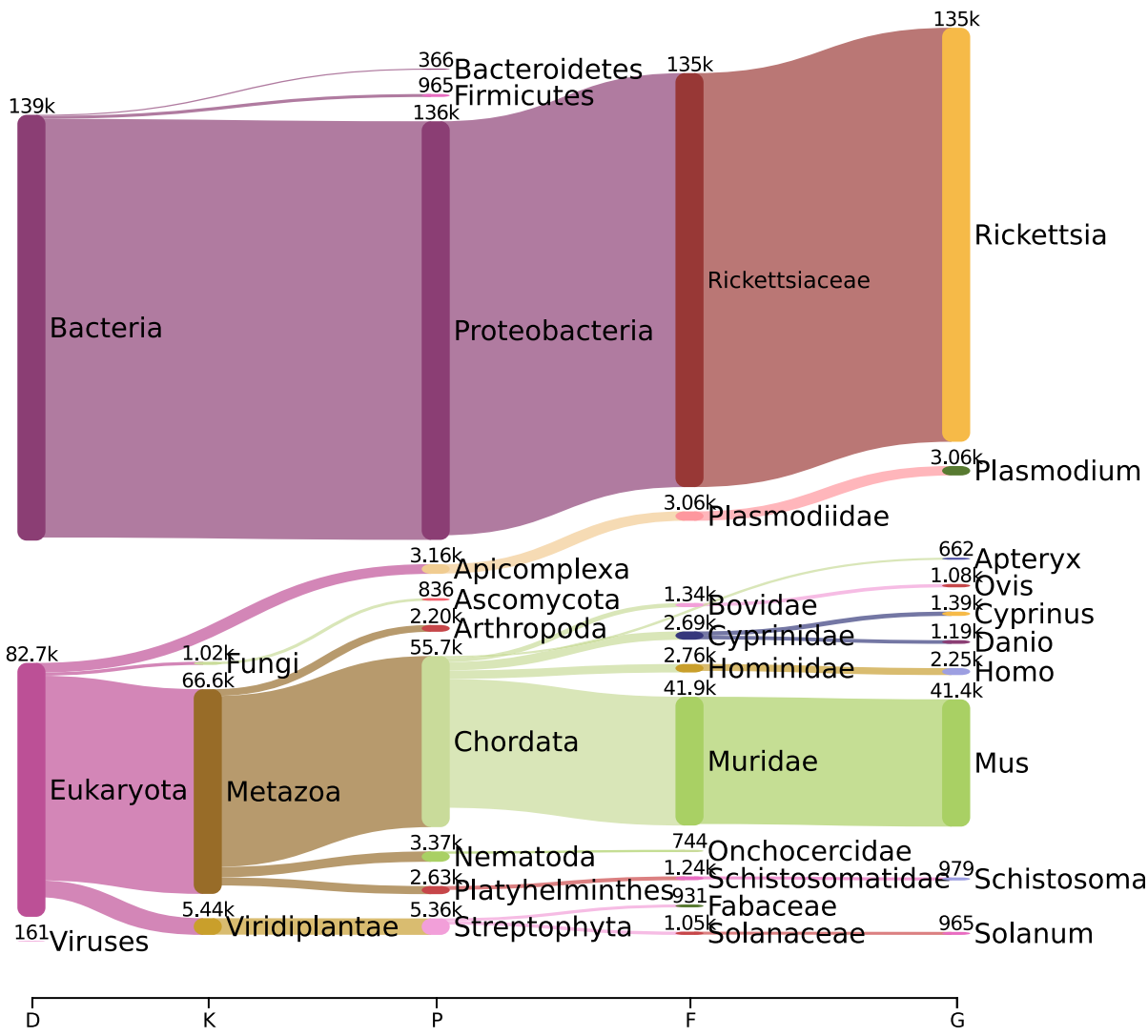

FIGURE 1. Pavian visualization showing the proportions of Oxford Nanopore Technologies' reads assigned up to the genus level ${ }^{32}$. This figure appears in color at www.ajtmh.org. 


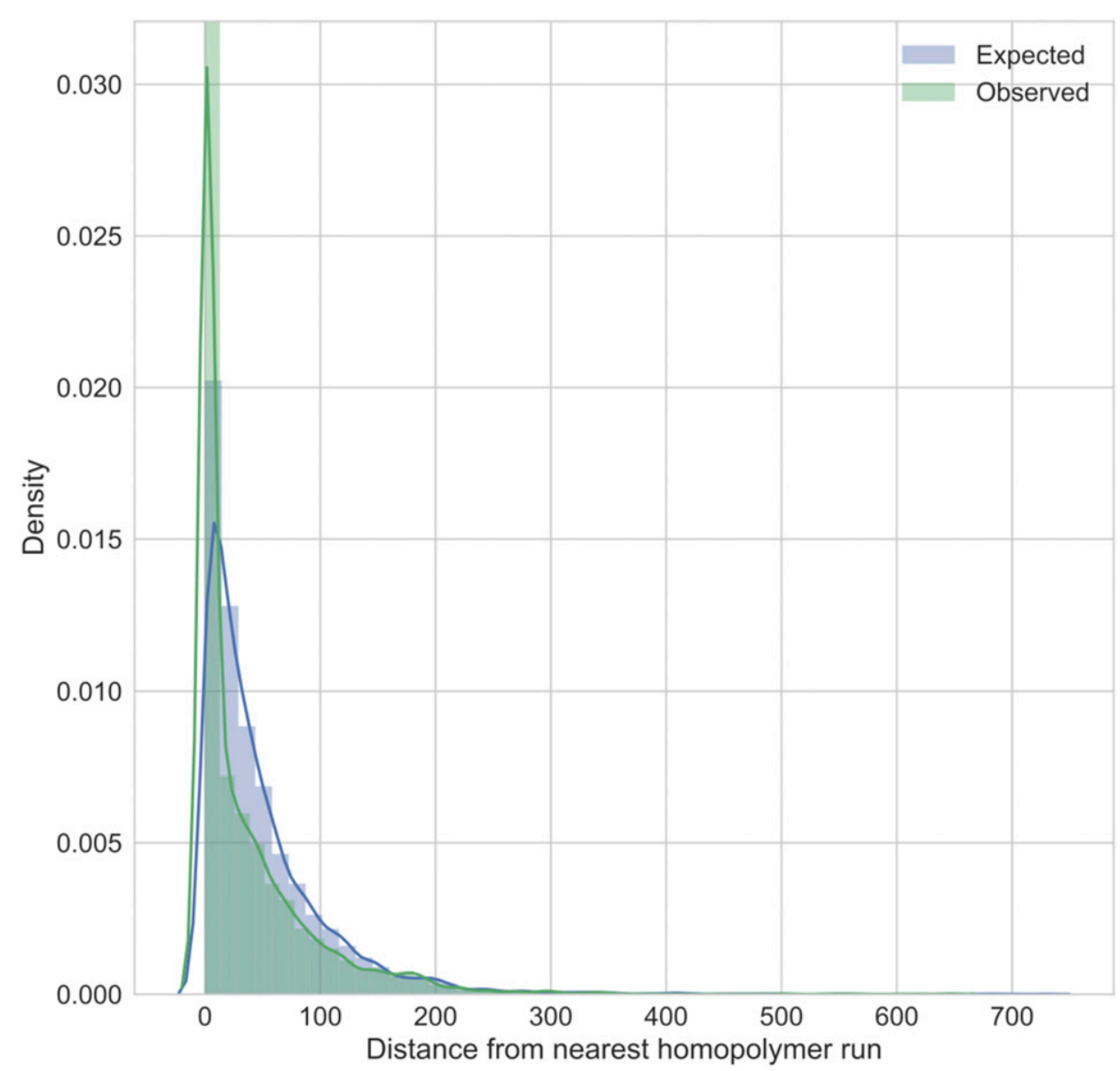

FIGURE 2. The observed distance from a homopolymer run for the errors corrected by Pilon in the draft genome, compared with the expected distribution if errors were randomly distributed across the genome. This figure appears in color at www.ajtmh.org.

search. These contigs are identified by their low coverage depth relative to $R$. typhi contigs. After removal of contaminating mouse contigs, the Unicycler assembly has eight contigs with a total length of $1,111,455 \mathrm{bp}$.

Furthermore, BUSCO was used to quantitatively measure the genome completeness of our assemblies based on a set of 48 conserved single-copy genes from the OrthoDB v9 database $^{33}$ (Table 2). None of the genes were found in the initial Canu assembly. Inspection of the TBLASTN results shows that while there were hits to many of the conserved genes, they fall below the threshold where BUSCO will call them as present. After polishing with Nanopolish using ONT data only, $14.2 \%$ of the genes could be found as a complete copy, with a further $32.4 \%$ found as fragmented genes. Two rounds of
Pilon polishing improve this to $88.5 \%$ of genes found as complete copies. Running BUSCO on the Wilmington reference strain gives the same number of complete genes, suggesting this is the complete set of the orthologous reference genes that exists in $R$. typhi. The Unicycler assembly also contains this complete set of genes.

The final polished genome assembly is 1,111,939 bp, extremely similar to the three available genomes, which range from $1,111,496$ to $1,112,957 \mathrm{bp}$ in length (Table 1). Alignment of the four whole genomes in Mauve ${ }^{34}$ shows that the genomes are colinear and that no rearrangements have taken place between strains (Supplemental Figure 3).

The TM2540 genome contained 866 predicted genes, very close to the 865 genes predicted in all other strains. Roary was

TABLE 2

Proportion of BUSCO bacterial gene set found as complete genes, fragmented genes, or missing for different assemblies of the TM2540 strain, and the reference Wilmington assembly

\begin{tabular}{lrr}
\hline & Complete (\%) & Missing (\%) \\
\hline Canu only & 0.00 & 3.40 \\
Canu + Nanopolish & 14.20 & 32.40 \\
Canu + Nanopolish + Pilon (1 round) & 85.10 & 2.70 \\
Canu + Nanopolish + Pilon (2 rounds) & 83.40 & 0.70 \\
Canu + Nanopolish + Pilon (3 rounds) & 88.50 & 0.70 \\
Canu + Nanopolish + Pilon (4 rounds) & 88.50 & 0.70 \\
Unicycler (Illumina only) & 88.50 & 0.70 \\
Reference genome (Wilmington) & 88.50 & 10.80 \\
\hline
\end{tabular}


used to define the core genome (genes present in all strains at $90 \%$ identity) of the four $R$. typhi strains, which included 863 genes. Of the five accessory genes not found in all strains, two were found in three of the four strains and three were unique to a single strain. Two of the genes not found in all samples were the same gene (RT_RS01750 in strain Wilmington), but clustered separately because of a 150-bp insertion in the B991CWPP strain that caused the sequence to fall below the $90 \%$ identity threshold to cluster with the other three strains. In two of the five accessory genes, a single base insertion in one strain has created a premature stop codon, splitting a gene into two sequences in that strain (RT_RS02175 in strain Wilmington, and TM2540_RS03925 in strain TM2540). In the final case, the gene is present in three strains next to a duplicate pseudogene which has lost the start and end of the gene, whereas in the fourth strain, a deletion has removed the end of the pseudogene and the start of the gene, leaving two incomplete and presumably inactive copies of the gene (RT_ RS03370 in strain Wilmington). All five variably present genes are annotated as hypothetical proteins in all strains.

SNPs and indels in the four genomes were called from the alignment of the four reference genomes. We found 32 SNPs and 30 small indels (size range 1-782 bp). A full listing of the variants and their location in the Wilmington strain genome is given in Supplemental Table 2. Table 3 shows the number of differences between strains (counting each indel as a single event).

One of the small indels is in the RARP2 gene, which was previously noted to have a variable number of ankyrin repeats. Our new strain TM2540 has the same variant previously found in B9991CWPP and TH1527 and differs from the reference Wilmington strain. We also note a deletion in penicillin-binding protein 4 ( $p b p E)$ in TM2540 which leads to a truncated protein product. Variants are also present in the surface cell antigen (sca) gene families, with SNPs in sca1 and sca3 and indels in sca2 and sca4.

We compared the results of the final polished assembly to the best assemblies generated using Illumina data alone (filtered Unicycler assembly) or ONT data alone (Canu + Nanopolish assembly). The Illumina assembly has 866 predicted genes, and the core genome using this assembly has 862 core genes and seven accessory genes. This includes one gene unique to the Illumina assembly, which is part of a gene split into two pieces at a contig boundary. The ONT assembly has 1,745 predicted genes, but this number is inflated by a number of genes which have been split into multiple pieces likely because of sequence errors introducing premature stop codons in the sequence, and the core genome using this assembly has only 774 genes.

\section{DISCUSSION}

WGS of pathogens is an increasingly important tool in both research and clinical settings, whose use has increased over

TABLE 3

Pairwise differences between strains based on the whole genome alignment

\begin{tabular}{lcccc}
\hline & Wilmington & B9991CWPP & TH1527 & TM2540 \\
\hline Wilmington & 0 & & & \\
B9991CWPP & 27 & 0 & & \\
TH1527 & 18 & 21 & 0 & \\
TM2540 & 40 & 45 & 36 & 0 \\
\hline
\end{tabular}

the last decade as its cost has decreased and availability improved. However, the initial investment in sequencing and computing equipment and the technical expertise needed to produce and analyze data remain a barrier to its introduction into resource-poor settings. Although our research laboratory can perform molecular diagnostics and cell culture, to date there has been no sequencing capacity, necessitating the slow and costly shipment of samples to other countries to undertake sequencing projects. The portable nature of the MinION sequencer has allowed the first bacterial WGS to be performed in Laos. We were able to run all steps of our bioinformatic analysis (except Centrifuge) on a laptop computer (MacBook Pro 2017) without continuous Internet access, demonstrating that sequencing and analysis can take place in relatively remote settings.

Murine typhus is an important and severely neglected tropical and subtropical disease of worldwide distribution. In Vientiane, an estimated $10 \%$ of non-malarial fevers in adult inpatients are caused by $R$. typhi. ${ }^{35}$ Surprisingly, for a disease to which many millions of people are potentially exposed, there are only three published whole genome sequences, of which the last human isolate dates back more than 50 years.

The $R$. typhi genome is $1.1 \mathrm{Mb}$ and contains almost no repeats, which makes genome assembly for this species relatively simple. Running Canu with the default ONT parameters was sufficient to assemble the genome into a single contig, and we polished the assemblies using both Nanopolish, to use the signal-level data from ONT sequencing, and Pilon, to use the extra information available from Illumina sequencing with its lower error rate. Although its synteny with other genomes suggests that there are no large misassemblies in our genome, the two methods of polishing corrected many small SNPs and indel errors in the ONT only draft genome. Thus, even for relatively simple bacterial genomes, a combination of ONT with data from another technology with a low error rate is currently necessary to produce an accurate sequence. In the case of $R$. typhi, the strains are so closely related that distinguishing between them requires a highly accurate sequence, and using ONT data alone, the sequencing errors are more numerous than the true differences between strains. Removing regions of the genome close to homopolymer runs would remove a large number of positions with errors by filtering out a comparatively small amount of the genome, but would still leave over 2,000 more errors than true differences. Oxford Nanopore Technologies data alone may suffice for applications which can work with sequences which still retain some base pair-level errors, such as determining large-scale genome rearrangements, ${ }^{36}$ determining the species of an unknown sample, ${ }^{37,38}$ or detecting antimicrobial resistance genes. ${ }^{39}$ Increases in sequencing accuracy, combined with bioinformatics advances in assembly and polishing software, may allow for the future use of ONT data alone to give complete and accurate sequences, increasing the applications for this device.

Comparative analysis of the four $R$. typhi genomes shows very little variation between strains. Very few genes are variably present between strains, and the gene order is completely conserved. Although these four samples were collected from humans and a rodent over a long timeframe, we see only 23 SNPs between the two most divergent strains, no large-scale genome rearrangements, and very few indels. The 
low number of differences suggests that WGS may not be a useful tool to analyze the relationships between strains of $R$. typhi, as there may be few differences between strains and no recent shared ancestry. Recent phylogenomic studies of multiple rickettsial species have identified very little divergence within species. ${ }^{40,41}$ Despite this small amount of variation, there are SNPs and indels which are private to each strain and could potentially form the basis of a molecular typing scheme to differentiate lineages of $R$. typhi. There are also variants in four of the five sca family genes present in $R$. typhi, which are important in adhesion and invasion of mammalian cells, ${ }^{42}$ and variation may be expected in these genes because of the selective pressure they are under as cell surface antigens; however, they are also large proteins and may be more likely to accumulate variants because of their size.

Some limitations remain on the use of ONT's MinION platform. Currently, individual flow cells remain costly at USD500USD $900,{ }^{43}$ depending on the number purchased. However, the cost per sample can be reduced because multiplex sequencing now allows for multiple bacterial strains to be sequenced on the same flow cell. ${ }^{44}$ Ongoing improvements in the technology continue to increase overall output and per-run capacity. Costs for setting up MinION sequencing facilities such as an appropriate laptop computer, shipping reagents, and flow cells are expected to be low. Shipping DNA or RNA at $-80^{\circ} \mathrm{C}$ may cost more than USD1,000 per shipment and frequently requires supporting documentation and permissions. These costs remain a fraction of those required to set up and maintain nonportable sequencing platforms. Novel technologies are likely to improve access to sequencing when computational resources and support are limited and allow more cost-efficient sequencing of single samples. ${ }^{45}$

MinION instruments continue to be used in an everwidening set of challenging circumstances. The platform has been used to detect and monitor nosocomial tuberculosis in Zambia, ${ }^{46}$ to sequence Ebola and Zika virus in Guinea and Brazil $^{3,47}$ at field sites, to sequence amphibian DNA in montane rainforest in Tanzania, ${ }^{48}$ and for off-line use in the high Arctic and on the International Space Station. ${ }^{49-51}$ Here, we demonstrate the current applicability of the MinION to resource-poor settings where some laboratory infrastructure exists, but WGS capacity is unavailable. With concerns in some countries about the export of biological samples for WGS in other countries, MinION systems could facilitate countries without current WGS facilities to undertake such work.

Received May 20, 2019. Accepted for publication October 25, 2019.

Published online December 9, 2019.

Note: Supplemental tables and figures appear at www.ajtmh.org.

Acknowledgments: We are very grateful to Associate Professor Bounthaphany Bounxouei, past Director of Mahosot Hospital; the Director Manivanh Vongsouvath and staff of the microbiology laboratory, LOMWRU; the wards; Assistant Professor Chanphomma Vongsamphan, Director of Department of Health Care, Ministry of Health; and H.E. Professor Bounkong Syhavong, Minister of Health, Laos, for their help and support. We also thank Narongchai Tongyoo and Phonepasith Panyanouvong for growing the isolate in cell culture, and Duncan Parkes for assistance with performing base-calling.

Financial support: This study was supported by I. E.'s Wellcome Trust Research Training Fellowship (105731/Z/14/Z) and in part by Core Awards from the Wellcome Trust (090532/Z/09/Z and 203141/Z/16/Z). MTR and PNN are supported by the Wellcome Trust.
Authors' addresses: Ivo Elliott, Matthew T. Robinson, and Paul N. Newton, Lao-Oxford-Mahosot Hospital-Wellcome Trust Research Unit, Microbiology Laboratory, Mahosot Hospital, Vientiane, Lao PDR, and Nuffield Department of Medicine, Centre for Tropical Medicine and Global Health, University of Oxford, Oxford, United Kingdom, E-mails: ivo@tropmedres.ac, matthew.r@tropmedres.ac, and paul.newton@ tropmedres.ac. Elizabeth M. Batty, Nuffield Department of Medicine, Centre for Tropical Medicine and Global Health, University of Oxford, Oxford, United Kingdom, Wellcome Centre for Human Genetics, University of Oxford, Oxford, United Kingdom, and Mahidol-Oxford Tropical Medicine Research Unit, Faculty of Tropical Medicine, Mahidol University, Bangkok, Thailand, E-mail: elizabeth.b@tropmedres.ac. Damien Ming and Pruksa Nawtaisong, Lao-Oxford-Mahosot HospitalWellcome Trust Research Unit, Microbiology Laboratory, Mahosot Hospital, Vientiane, Lao PDR, E-mails: damien.ming@nhs.net and pruksa@gmail.com. Mariateresa de Cesare and Rory Bowden, Wellcome Centre for Human Genetics, University of Oxford, Oxford, United Kingdom, E-mails: decesare@well.ox.ac.uk and rbowden@well.ox.ac.uk.

This is an open-access article distributed under the terms of the Creative Commons Attribution (CC-BY) License, which permits unrestricted use, distribution, and reproduction in any medium, provided the original author and source are credited.

\section{REFERENCES}

1. National Human Genome Research Institute (NHGRI), 2019. The Cost of Sequencing a Human Genome. Available at: https:// www.genome.gov/about-genomics/fact-sheets/SequencingHuman-Genome-cost. Accessed November 14, 2017.

2. Jain M, Olsen HE, Paten B, Akeson M, 2016. The Oxford nanopore MinION: delivery of nanopore sequencing to the genomics community. Genome Biol 17: 239.

3. Quick J et al., 2016. Real-time, portable genome sequencing for Ebola surveillance. Nature 530: 228-232.

4. Loman NJ, Quick J, Simpson JT, 2015. A complete bacterial genome assembled de novo using only nanopore sequencing data. Nat Methods 12: 733-735.

5. Risse J, Thomson M, Patrick S, Blakely G, Koutsovoulos G, Blaxter M, Watson M, 2015. A single chromosome assembly of Bacteroides fragilis strain BE1 from Illumina and MinION nanopore sequencing data. GigaScience 4: 60.

6. Istace B et al., 2017. De novo assembly and population genomic survey of natural yeast isolates with the Oxford nanopore MinION sequencer. GigaScience 6: 1-13.

7. Jansen HJ et al., 2017. Rapid de novo assembly of the European eel genome from nanopore sequencing reads. Sci Rep 7: 7213.

8. Jain M et al., 2018. Nanopore sequencing and assembly of a human genome with ultra-long reads. Nat Biotechnol 36: 338-345.

9. Goodwin S, Gurtowski J, Ethe-Sayers S, Deshpande P, Schatz MC, McCombie WR, 2015. Oxford Nanopore sequencing, hybrid error correction, and de novo assembly of a eukaryotic genome. Genome Res 25: 1750-1756.

10. United Nations Development Programme, 2015. Human Development Index (HDI). Available at: http://hdr.undp.org/en/ content/human-development-index-hdi. Accessed November 14, 2017.

11. Speedtest, 2018. Speedtest Global Index. Available at: http:// www.speedtest.net/global-index. Accessed August 13, 2017.

12. Dumler JS, Walker DH, 2010. Rickettsia typhi (Murine Typhus). Mandell GL, Bennett JE, Dolin R, eds. Mandell, Douglas, and Bennett's Principles and Practice of Infectious Diseases, 7th edition. Philadelphia, PA: Churchill Livingstone, 2525-2528.

13. Tsioutis C, Zafeiri M, Avramopoulos A, Prousali E, Miligkos M, Karageorgos SA, 2017. Clinical and laboratory characteristics, epidemiology, and outcomes of murine typhus: a systematic review. Acta Trop 166: 16-24.

14. Azad AF, 1990. Epidemiology of murine typhus. Annu Rev Entomol 35: 553-569.

15. Civen R, Ngo V, 2008. Murine typhus: an unrecognized suburban vectorborne disease. Clin Infect Dis 46: 913-918.

16. Dittrich $S$ et al., 2015. Orientia, rickettsia, and leptospira pathogens as causes of CNS infections in Laos: a prospective study. Lancet Glob Health 3: e104-e112. 
17. Newton PN et al., 2018. A prospective, open-label, randomized trial of doxycycline versus azithromycin for the treatment of uncomplicated murine typhus. Clin Infect Dis 68: 738-747.

18. McLeod MP et al., 2004. Complete genome sequence of Rickettsia typhi and comparison with sequences of other rickettsiae. J Bacteriol 186: 5842-5855.

19. Maxcy KF, 1929. Endemic typhus fever of the southeastern United States: reaction of the Guinea pig. Public Health Rep 44: 589-600.

20. Institute USDoEJG, 2014. Rickettsia typhi TH1527. Available at: https://gold.jgi.doe.gov/project?id=11636. Accessed November 14, 2017.

21. Sankasuwan $V$, Pongpradit $P$, Bodhidatta $P$, Thonglongya $K$, Winter PE, 1969. Murine typhus in Thailand. Trans $R$ Soc Trop Med Hyg 63: 639-643.

22. Institute USDoEJG, 2014. Rickettsia typhi B9991CWPP. Available at: https://gold.jgi.doe.gov/project?id=11634. Accessed November 14, 2017.

23. Jiang J, Chan TC, Temenak JJ, Dasch GA, Ching WM, Richards AL, 2004. Development of a quantitative real-time polymerase chain reaction assay specific for Orientia tsutsugamushi. Am J Trop Med Hyg 70: 351-356.

24. Kim D, Song L, Breitwieser FP, Salzberg SL, 2016. Centrifuge: rapid and sensitive classification of metagenomic sequences. Genome Res 26: 1721-1729.

25. Koren S, Walenz BP, Berlin K, Miller JR, Bergman NH, Phillippy AM, 2017. Canu: scalable and accurate long-read assembly via adaptivek-mer weighting and repeat separation. Genome Res 27: 722-736.

26. Li H, 2013. Aligning Sequence Reads, Clone Sequences and Assembly Contigs with BWA-MEM. Available at: https://arxiv.org/ abs/1303.3997. Accessed January 30, 2018.

27. Walker BJ et al., 2014. Pilon: an integrated tool for comprehensive microbial variant detection and genome assembly improvement. PLoS One 9: e112963.

28. Simao FA, Waterhouse RM, loannidis $\mathrm{P}$, Kriventseva EV, Zdobnov EM, 2015. BUSCO: assessing genome assembly and annotation completeness with single-copy orthologs. Bioinformatics 31: 3210-3212.

29. Wick RR, Judd LM, Gorrie CL, Holt KE, 2017. Unicycler: resolving bacterial genome assemblies from short and long sequencing reads. PLoS Comput Biol 13: e1005595.

30. Seemann T, 2014. Prokka: rapid prokaryotic genome annotation. Bioinformatics; 30: 2068-2069.

31. Page AJ, Cummins CA, Hunt M, Wong VK, Reuter S, Holden MT, Fookes M, Falush D, Keane JA, Parkhill J, 2015. Roary: rapid large-scale prokaryote pan genome analysis. Bioinformatics 31: 3691-3693.

32. Breitwieser FP, Salzberg SL, 2019. Pavian: interactive analysis of metagenomics data for microbiomics and pathogen identification. Bioinformatics btz715.

33. Kriventseva EV, Tegenfeldt F, Petty TJ, Waterhouse RM, Simao FA, Pozdnyakov IA, loannidis P, Zdobnov EM, 2015. OrthoDB v8: update of the hierarchical catalog of orthologs and the underlying free software. Nucleic Acids Res 43: D250-D256.

34. Darling AE, Mau B, Perna NT, 2010. progressiveMauve: multiple genome alignment with gene gain, loss and rearrangement. PLoS One 5: e11147.
35. Phongmany $S$ et al., 2006. Rickettsial infections and fever, Vientiane, Laos. Emerg Infect Dis 12: 256-262.

36. McGinty RJ, Rubinstein RG, Neil AJ, Dominska M, Kiktev D, Petes TD, Mirkin SM, 2017. Nanopore sequencing of complex genomic rearrangements in yeast reveals mechanisms of repeatmediated double-strand break repair. Genome Res 27: 2072-2082.

37. Parker J, Helmstetter AJ, Devey D, Wilkinson T, Papadopulos AST, 2017. Field-based species identification of closely-related plants using real-time nanopore sequencing. Sci Rep 7: 8345.

38. Batovska J, Lynch SE, Rodoni BC, Sawbridge TI, Cogan NO, 2017. Metagenomic arbovirus detection using MinION nanopore sequencing. J Virol Methods 249: 79-84.

39. Schmidt $\mathrm{K}$ et al., 2017. Identification of bacterial pathogens and antimicrobial resistance directly from clinical urines by nanopore-based metagenomic sequencing. J Antimicrob Chemother 72: 104-114.

40. Hagen R, Verhoeve VI, Gillespie JJ, Driscoll TP, 2018. Conjugative transposons and Their cargo genes vary across natural populations of Rickettsia buchneri infecting the tick ixodes scapularis. Genome Biol Evol 10: 3218-3229.

41. Diop A, Raoult D, Fournier PE, 2019. Paradoxical evolution of rickettsial genomes. Ticks Tick Borne Dis 10: 462-469.

42. Sears KT, Ceraul SM, Gillespie JJ, Allen ED Jr., Popov VL, Ammerman NC, Rahman MS, Azad AF, 2012. Surface proteome analysis and characterization of surface cell antigen (Sca) or autotransporter family of Rickettsia typhi. PLoS Pathog 8: e1002856.

43. Oxford Nanopore Technologies, 2018. Available at: https:// nanoporetech.com/products\%20-\%20modal=comparison. Accessed March 23, 2018.

44. Wick RR, Judd LM, Gorrie CL, Holt KE, 2017. Completing bacterial genome assemblies with multiplex MinION sequencing. Microb Genom 3: e000132.

45. Oxford Nanopore Technologies, 2018. Available at: https:// nanoporetech.com/products/flongle. Accessed May 09, 2018.

46. Bates M, Polepole P, Kapata N, Loose M, O'Grady J, 2016. Application of highly portable MinION nanopore sequencing technology for the monitoring of nosocomial tuberculosis infection. Int J Mycobacteriol 5 (Suppl 1): S24.

47. Faria NR, Sabino EC, Nunes MR, Alcantara LC, Loman NJ, Pybus OG, 2016. Mobile real-time surveillance of Zika virus in Brazil. Genome Med 8: 97.

48. Menegon $M$, Cantaloni $C$, Rodriguez-Prieto A, Centomo $C$, Abdelfattah A, Rossato M, Bernardi M, Xumerle L, Loader S, Delledonne M, 2017. On site DNA barcoding by nanopore sequencing. PLoS One 12: e0184741.

49. Johnson SS, Zaikova E, Goerlitz DS, Bai Y, Tighe SW, 2017. RealTime DNA sequencing in the antarctic dry valleys using the Oxford nanopore sequencer. J Biomol Tech 28: 2-7.

50. Goordial J, Altshuler I, Hindson K, Chan-Yam K, Marcolefas E, Whyte LG, 2017. In situ field sequencing and life detection in remote ( 79 degrees $26^{\prime} \mathrm{N}$ ) Canadian high arctic permafrost ice wedge microbial communities. Front Microbiol 8: 2594.

51. Castro-Wallace SL et al., 2017. Nanopore DNA sequencing and genome assembly on the international space station. Sci Rep 7: 18022. 